\title{
Gastrointestinale Onkologie - Therapiestrategien der Zukunft
}

Für verschiedene Tumorentitäten des Gastrointestinaltrakts hat sich die Prognose in den letzten 20 Jahren deutlich verbessert. Es wurden neue zielgerichtete Therapien entwickelt, und durch die Charakterisierung von definierten Subgruppen kann die Sensitivität bzw. Resistenz gegenüber bestimmten Therapiekonzepten vorhergesagt werden. Den entscheidenden Einfluss auf die Prognose des Kolonkarzinoms hatte allerdings die Einführung der Vorsorgekoloskopie. Die Zukunft liegt vor allem in der primären und sekundären Prävention von gastrointestinalen Tumorerkrankungen.

Das Pankreaskarzinom hat die schlechteste Prognose aller gastrointestinalen Tumorerkrankungen. Beispielhaft an dieser Tumorerkrankung möchte ich mögliche Zukunftsstrategien erläutern. Die schlechte Prognose liegt einerseits an der Diagnosestellung Pankreaskarzinom zu einem späten Zeitpunkt, andererseits aber vor allem auch an seinen biologischen Eigenschaften. Während lange Zeit die zellautonomen Mechanismen der Tumorzellen im Fokus der Entwicklung von neuen Therapiestrategien standen, ist heutzutage klar, dass diese allein der Komplexität von Tumorerkrankungen nicht gerecht werden. Insbesondere das Pankreaskarzinom ist neben besonderen zellautonomen Eigenschaften auch durch „extreme“ nichtzellautonome Eigenschaften charakterisiert. Die Summe dieser biologischen Eigenschaften („unique features“) charakterisiert die Aggressivität dieser Tumorerkrankung, stellt aber auch Zielstrukturen für neue Therapiekonzepte dar.

\section{Therapiestrategien für die Zukunft}

\section{Definition molekularer Subgruppen}

Bisherige Therapieprotokolle weisen große Unterschiede im Ansprechen und im Gesamtüberleben auf. Zur Optimierung ist eine bessere Stratifizierung erforderlich. Dies gelingt einerseits durch die Charakterisierung struktureller Genveränderungen (KRAS, NTRK und BRACA2). Ande- rerseits kann eine weitere Differenzierung auf der Transkriptom-Ebene durch RNASequenzieranalysen erfolgen [1,2]. Beim Pankreaskarzinom kristallisieren sich zwei unterschiedliche Subgruppen mit unterschiedlichen Vulnerabilitäten gegenüber Chemotherapieprotokollen heraus. Des Weiteren weisen Pankreaskarzinomzellen eine hohe Plastizität auf, d.h. Tumorzellen können schnell durch epigenetische Regulationsmechanismen sekundäre Resistenzen gegen Therapeutika entwickeln.

\section{Screens für Vulnerabilitäten}

Die Hochdurchsatzsequenzierung hat zahlreiche strukturelle Genveränderungen auch beim Pankreaskarzinom identifiziert. Diese deskriptiven Daten erlauben nicht die Unterscheidung von Treibermutationen und begleitenden Genveränderungen. Dies erfordert funktionelle Assays z. B. mithilfe der CRISPR/Cas9-Technologie. Insbesondere synthetische Letalität stellt einen effektiven, nebenwirkungsarmen und innovativen Therapieansatz dar. Zwei Gene befinden sich in einer synthetisch letalen Interaktion, wenn der Defekt eines dieser Gene toleriert wird, während der simultane Defekt beider Gene letal ist. So können Tumorzellen, die bereits einen Genedefekt aufweisen (Mutation oder differentielle Expression), gezielt durch Hemmung eines zweiten Gens (synthetisch letaler Interaktionspartner) eliminiert werden, während gesunde Zellen ohne diesen Gendefekt weitestgehend unbeschadet bleiben.

\section{Stroma als therapeutische Zielstruktur}

Bei der Mehrzahl der Pankreaskarzinome finden sich mehr Stromazellen und extrazelluläre Matrix als Tumorzellen. Das Stroma wirkt als mechanische Barriere, es erschwert das Eindringen von Immunzellen in den Tumor, schränkt die vaskuläre Perfusion ein und damit das Erreichen von effektiven therapeutischen Dosen im Tumor. Die Fibroblasten im Tumor scheinen eine protektive Rolle zu spielen. Die therapeutische Hemmung der desmoplastischen Reaktion induziert aggressivere Tumoren. Die immunsuppressiven Elemente des Stromas werden derzeit als therapeutische
Zielstrukturen in klinischen Studien evaluiert. Die molekularen Grundlagen der Heterogenität des Stromas sind allerdings bislang noch weitgehend unklar. Des Weiteren lässt sich bei allen Pankreaskarzinomen eine perineurale Tumorinvasion nachweisen. Die neuro-tumorale Interaktion stellt damit eine weitere interessante therapeutische Zielstruktur dar.

\section{Paraneoplastische Prozesse}

Bereits kleine Pankreaskarzinome induzieren einen raschen Gewichtsverlust durch Kachexie und Sarkopenie. Die zugrunde liegenden katabolen Mechanismen sind völlig unklar. Mehr als 85\% der Pankreaskarzinompatienten weisen bei Diagnosestellung zumindest eine Glukose-ToleranzStörung oder einen Diabetes mellitus auf. Die paraneoplastische Ursache dieser metabolischen Störung wird letztlich nicht gut verstanden. Der BMI ist ein unabhängiger Prognosefaktor beim Pankreaskarzinom, sodass hier eine Korrektur die Prognose einer Mehrzahl von Pankreaskarzinompatienten deutlich verbessern würde.

Patienten mit Pankreaskarzinom weisen die höchste Thromboserate aller Tumorerkrankungen auf. Thrombosen sind mit einer schlechteren Prognose assoziiert. Die prophylaktische Antikoagulation wird derzeit in großen Studien untersucht.

\section{Workflow}

Um die molekularen und funktionellen Eigenschaften von Pankreaskarzinomen individuell untersuchen zu können, wird Turmormaterial durch endosonografische Punktion vor Therapiebeginn gewonnen. Im Labor werden daraus Organoide (MiniOrgane) kultiviert. Durch die molekulare Charakterisierung erfolgt die Einteilung in spezifische Subgruppen. In funktionellen Untersuchungen können dann individuelle Vulnerabilitäten gegenüber Chemotherapie-Protokollen und neuen Medikamenten in den Organoiden getestet werden [3].

Insgesamt stellt die Translation der individualisierten Therapie des Pankreaskarzinoms eine große Herausforderung dar [4]. 
Interessenkonflikt

Der Autor gibt an, dass kein Interessenkonflikt besteht.

\section{Autor}

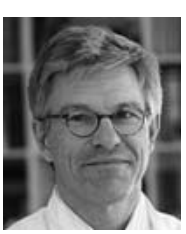

\section{Roland M. Schmid}

Prof. Dr. med., Direktor der II. Medizinischen Klinik u. Poliklinik, Technische Universität München, Klinikum rechts der Isar, München, Deutschland
Prof. Dr. med. Roland M. Schmid Direktor der II. Medizinischen Klinik u. Poliklinik

Technische Universität München Klinikum rechts der Isar Ismaninger Straße 22

81675 München

Deutschland

direktion.med2@mri.tum.de
Literatur

[1] Eser S, Schnieke A, Schneider G et al. Oncogenic KRAS signalling in pancreatic cancer. $\mathrm{Br}$ J Cancer 2014; 111: 817-822. doi:10.1038/bjc.2014.215.

[2] Mueller S, Engleitner T, Maresch et al. Evolutionary routes and KRAS dosage define pancreatic cancer phenotypes. Nature 2018; 554: 62-68. doi:10.1038/nature25459

[3] Dantes Z, Yen HY, Pfarr $N$ et al. Implementing cell-free DNA of pancreatic cancer patient-derived organoids for personalized oncology. JCl Insight 2020; 5: e137809. doi:10.1172/jci.insight.137809

[4] Falcomatà C, Schneider G, Saur D. Personalizing KRAS-mutant allele-specific therapies. Cancer Discov 2010; 10: 23-25. doi:10.1158/2159-8290.CD-19-1261. 